\begin{tabular}{|c|l|}
\hline Title & A erodynamic stability of road vehicles in dynamic pitching motion \\
\hline Author(s) & Cheng, S.Y.; T subokura, M.; Okada, Y.; Nouzawa, T.; Nakashima, T.; Doh, D.H. \\
\hline Citation & $\begin{array}{l}\text { Journal of Wind Engineering and Industrial A erodynamics, 122, 146-156 } \\
\text { https://doi.org/10.1016/.jweia.2013.06.010 }\end{array}$ \\
\hline Issue Date & 2013 11 \\
\hline Doc URL & http://hdl.handle.net/2115/53461 \\
\hline Type & article (author version) \\
\hline File Information & manuscript.pdf \\
\hline
\end{tabular}

Instructions for use 


\title{
Aerodynamic stability of road vehicles in dynamic pitching motion
}

Cheng, S.Y. ${ }^{\mathrm{a}, \mathrm{b}}$, Tsubokura, M. ${ }^{\text {a,c* }}$, Nakashima, T. ${ }^{\mathrm{d}}$, Nouzawa, T. ${ }^{\mathrm{e}}$, Okada, Y. ${ }^{\mathrm{e}}$ and Doh, D.H.

${ }^{a}$ Graduate School of Engineering, Hokkaido University, Kita-13, Nishi-8, Kita-ku, Sapporo, 060-8628, Japan.

${ }^{b}$ Faculty of Mechanical Engineering, Universiti Teknikal Malaysia Melaka, Hang Tuah Jaya, 76100 Durian Tunggal, Melaka, Malaysia.

${ }^{c}$ RIKEN Advanced Institute for Computatonal Science, 7-1-26 Minatojima-MinamiMachi,Chuo-ku, Kobe, Hyogo 650-0047, Japan.

${ }^{d}$ Graduate School of Engineering, Hiroshima University, 1-4-1 Higashi-Hiroshima, Hiroshima, 7398527, Japan.

${ }^{e}$ Vehicle Testing \& Research Department, Mazda Motor Corporation, Aki Gun, Hiroshima, 7308670, Japan.

${ }^{f}$ Division of Mechanical Energy Systems Engineering, Korea Maritime University, Yeongdo-ku, Busan,606-791, Korea.

*Corresponding author. Tel. \& Fax: +81 11-706-6723, E-mail:

mtsubo@eng.hokudai.ac.jp

\begin{abstract}
The main object of the present study is to investigate numerically the mechanism of aerodynamic damping of pitching oscillation in sedan-type vehicles. The transient numerical solver employed is based on the Large Eddy Simulation (LES) method. Whilst, the Arbitrary Lagrangian-Eulerian (ALE) method was used to realize the vehicle motion during dynamic pitching and fluid flow coupled simulations. Validation of the numerical method was done by comparing the flow structures obtained from the LES to the corresponding flow structures observed in the wind tunnel measurements. Two vehicle models with basic sedan-type automobile shape were created to study the influences of upper body geometry on the aerodynamic pitching stability of sedan-type vehicle. In addition, the credibility of modeling of automotive aerodynamics by simple bluff body models was verified. For the sedan-type models investigated, the trailing vortices that shed from the A-pillar and C-pillar edges were found to produce the opposite tendencies on how they affect the aerodynamic pitching stability of the models. In particular, the vortex shed from the A-pillar edge tended to enhance the pitching oscillation, while the vortex shed from the C-pillar edge tended to suppress it. Hence, the vehicle with rounded A-pillar and angular C-pillar exhibited a higher aerodynamic damping than the vehicle with the opposite A- and C-pillars configurations. The aerodynamic damping mechanism has been proposed based on the results of flow visualization on the phase-averaged flow properties.
\end{abstract}

Keywords: LES; aerodynamics; automobile; stability; pitching; damping; vortex; transient; sedan; pillar

\section{Introduction}

In bluff body flow, aerodynamic loading plays an important role in the behavior of a solid object immersed in a flow. The behavior can be assessed by identifying the characteristic of a parameter termed aerodynamic damping. In fact, this parameter has long been recognized in many areas of civil engineering application such like in transmission line system (e.g. Davenport, 1988; Momomura et al., 1997), building structure (e.g. Daw and Davenport, 1989; Murakawa et al., 1996; Watanabe et al, 1997), cable-stayed bridge (e.g. Virlogeux, 1998; Macdonald, 2002), wind turbines (e.g. Sinclair, 1994), etc. However, this is not the case in automotive engineering despite that road vehicles are also bluff bodies. Instead, the development of automotive aerodynamics has mainly been focused on the drag coefficient $C_{d}$. This is 
because fuel economy is one of the most important factors that determine the market situation of vehicle, especially in the wake of the 1973 oil crisis.

Other aerodynamic properties of vehicle may also be important, particularly the one related to handling and comfort performances. This is because when a vehicle is running on the road, especially at high speed, the flow structures generated around it may greatly influence its attitude. Today, this phenomenon has becoming more apparent because the advancement in engine power and better road condition have permitted higher running speed. In addition, the more stringent vehicle emissions standard has created a motivation for reducing the weight of vehicles, which makes them more susceptible to flowinduced effect.

To consider the stability issues, several assessment methods that take into consideration the dynamic motion of vehicle have been proposed in the literature. These methods rely on either drive test (e.g. Howell and Le Good, 1999; Okada et al., 2009) or wind tunnel measurement (e.g. Aschwanden et al., 2006). The applicability of the methods is bounded by their inherent limitations. For instance, the former can only be performed after a development mule is produced, while the latter requires a complex test rig to manipulate the vehicle motion for a dynamic assessment. In addition, due to limited numbers of probe that can be attached to the test vehicle without altering the surrounding flow, drive test and wind tunnel measurement provide very limited flow information about the test. The lack of flow information could impede detailed flow analysis which is needed to identify the physical mechanism (e.g. Okada et al., 2009).

To overcome these limitations, therefore, we have developed in the earlier phase of the study a numerical method which can resolve the spatial and temporal details of the flow properties, for the assessment of vehicle aerodynamic stability performance in a transient driving situation (interested reader is referred to Nakashima et al. (2009) and Cheng et al. $(2011,2012)$ ). In regard to the aerodynamic stability performance of vehicles, most studies reported in the literature were focus on yawing. Little effort has been made on pitching, despite its negative influence on drive comfort, control, and safety. Recently, Okada et al. (2009) has found that a small change in the upper body geometry of sedan-type vehicle can result in severe pitching oscillation problem. However, the road test and wind tunnel measurements employed in the study had provided limited flow information for identifying the mechanism. Hence, we attempted to address this issue by employing a numerical method.

In a real vehicle, the body shape configuration is complicated due to many projected body parts. The flow structures generated by these body parts can interfere between one another and render the results of investigation inconclusive. To overcome this problem, we simplified the vehicle shape by employing the models with simple bluff body shape in the earlier phase of the study (Nakashima et al., 2009; Cheng et al., 2011, 2012). This enables the isolation of the subject of investigation, i.e. the influence of upper body geometry on aerodynamic pitching stability, from the interference of other body parts. Thus conclusive outcomes can be derived.

In these investigations (Nakashima et al., 2009; Cheng et al., 2011, 2012), a coupling of ALE (Hirt et al., 1974) and LES methods had been employed to realize the vehicle body motion during fluid flow simulations, and to obtain the time-resolved results. As such, the studies were able to probe the dynamic response of the vehicle due to pitching oscillation. Thus, the stability performance of the vehicle models can be quantified based on the aerodynamic damping generated by them, which is depending on their body shape configuration. Moreover, the detailed flow information obtained from the numerical results has been exploited to verify the mechanism by which the aerodynamic forces affect the pitching stability of the models. Cheng et al. (2011) reported that the aerodynamic effect due to the trailing vortices shed from the front pillar (which correspond to the A-pillar in the case of sedan-type passenger car) tended to destabilize the model's pitching oscillation, while the aerodynamic effect due to the trailing vortices shed from the rear pillar (which correspond to the C-pillar) tended to stabilize it. Hence, the model with rounded front pillar and angular rear pillar exhibited a significantly higher aerodynamic damping than the model with the opposite pillar shape configurations (Cheng et al., 2012).

In this part of the study, we attempted to verify the credibility of the earlier approach, namely, modeling of real vehicle aerodynamics by simple body models. For this, we carried out similar investigation by the use of vehicle models with basic sedan-type vehicle shape. Then, we compared the results obtained from the two model types to verify the extent to which the results obtained from the simple bluff body models can be applied to real vehicle aerodynamics; particularly, in regard to aerodynamic pitching stability. In addition, we carried out extensive flow visualization based on the phase-averaged results to identify the mechanism of aerodynamic damping on the pitching oscillation of sedan-type vehicles. 


\section{Vehicle models}

We created two vehicle models with basic sedan-type vehicle shape for the investigation. The general geometrical features of the models are based on one of the vehicles used in the study by Okada et al. (2009), namely, Vehicle-A. Cheng et al. (2011, 2012) reported that the simple bluff body model with rounded front pillar and angular rear pillar shapes exhibited relatively large aerodynamic damping on the vehicle's pitching oscillation. Therefore, we adopted a rounder A-pillar shape and a more angular C-pillar shape for the basic sedan model that is expected to perform better in regard to aerodynamic stability. This is achieved by modifying the original pillar-shape configurations of Vehicle-A (see Fig. 1).

To focus on the influence of upper body, we simplified the shape of the models by adopting a flat underbody configuration. The models are at full scale, and have the same height $h$, width $w$, and length $l$ measurements of 4.7, 2.0, and $1.6 \mathrm{~m}$, respectively. The slant angles of A- and C-pillars are $30^{\circ}$ and $25^{\circ}$, respectively. For convenient in the discussions, the model with a more angular A-pillar and rounder Cpillar is designated "model A", while the other model is termed "model B", hereafter.
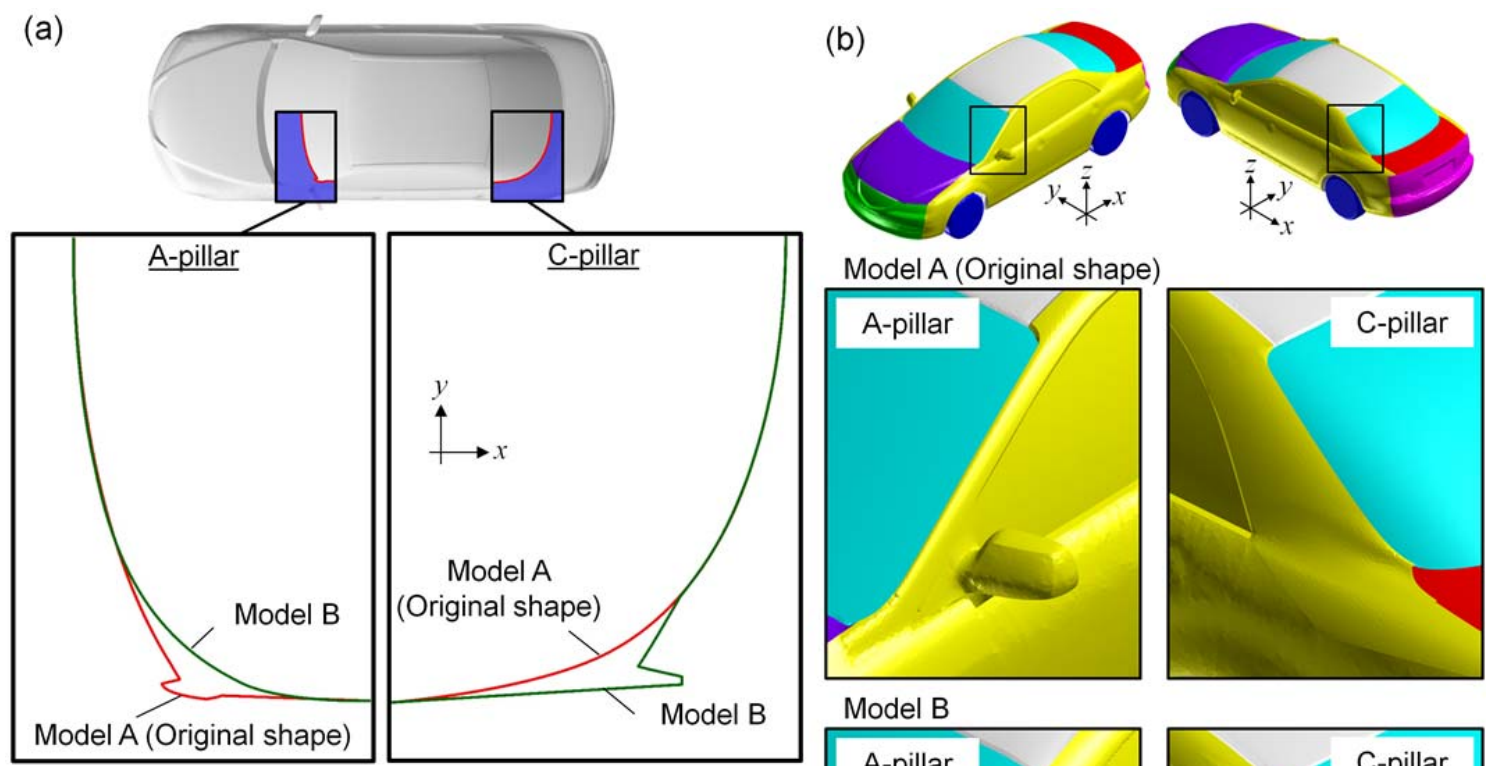

Model A (Original shape)
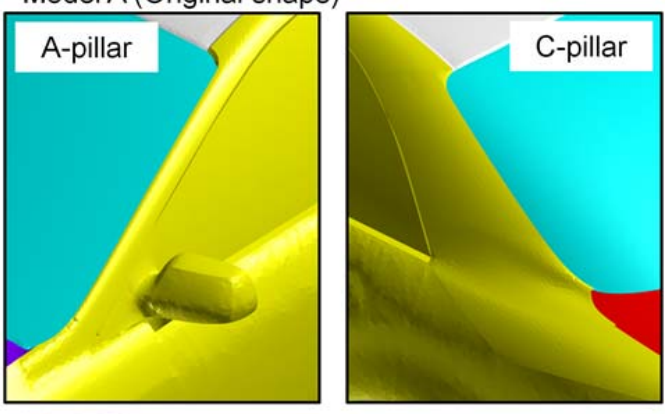

(c)

Bonnet Windshield
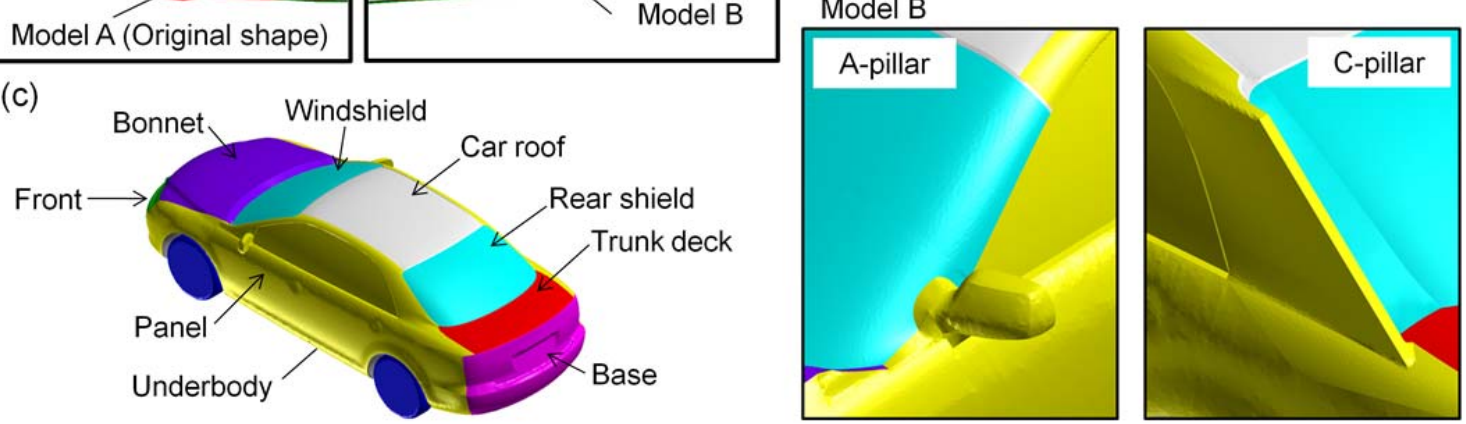

Fig. 1. (a) Profiles of A- and C-pillars, (b) Enlarged view of A- and C-pillar geometries, and (c) body part designations.

\section{Numerical Methods}

\subsection{Governing equations and discretization}

The LES solved the following spatially filtered continuity and Navier-Stokes equations: 


$$
\begin{aligned}
& \frac{\partial \bar{u}_{i}}{\partial x_{i}}=0 \\
& \frac{\partial \bar{u}_{i}}{\partial t}+\frac{\partial}{\partial x_{j}} \bar{u}_{i} \bar{u}_{j}=-\frac{\partial \bar{P}}{\partial x_{i}}+2 \frac{\partial}{\partial x_{j}}\left(v+v_{S G S}\right) \bar{S}_{i j} \\
& \bar{P}=p / \rho+\left(\overline{u_{l} u_{\jmath}}-\bar{u}_{i} \bar{u}_{j}\right) / 3
\end{aligned}
$$

where $u_{i}, p, \rho$, and $v$ are the $i$-th velocity component, pressure, density, and kinematic viscosity of the fluid. The over-bar ( ) indicates the spatially filtered quantity. The strain rate tensor $S_{i j}$ are defined as

$$
\bar{S}_{i j}=\frac{1}{2}\left(\frac{\partial \bar{u}_{j}}{\partial x_{i}}+\frac{\partial \bar{u}_{i}}{\partial x_{j}}\right)
$$

The subgrid-scale (SGS) eddy viscosity $v_{S G S}$ in equation (2) is modeled by the standard Smagorinsky model (Smagorinsky, 1963)

$$
v_{S G S}=\left(C_{s} f \Delta\right)^{2} \sqrt{2 \bar{S}_{i j} \bar{S}_{i j}}
$$

where $\Delta=\min \left(l_{y}, V^{1 / 3}\right), l_{y}$ is the distance between control volume $(\mathrm{CV})$ center and the nearest wall, and $V$ is the volume of the numerical mesh. As for the model coefficient $C_{s}$, we adopted a value of 0.15 . Meanwhile, we used the Van Driest damping function $f_{d}$ (Van Driest, 1956) to damp the effect of subgrid-scale eddy viscosity in the vicinity of solid boundary, which is defined as

$$
f_{d}=1-e^{\frac{-y^{+}}{25}}
$$

where $y^{+}$is the wall distance.

The governing equations were discretized by the vertex-centered unstructured finite volume method. The second-order central differencing scheme was applied for the spatial derivatives, and blending of $5 \%$ first-order upwind scheme for the convection term was exploited for numerical stability. Pressure-velocity coupling was preserved by the SMAC (Simplified Marker and Cell) algorithm (Amsden and Harlow, 1970).

For time advancement, we adopted the Euler implicit scheme. This is because an implicit scheme can accommodate larger time increment than an explicit one without causing numerical instability, especially in the case of a vehicle simulation in which the velocity and mesh size vary strongly. With larger permissible time increment, the scheme needs lesser time steps to cover the given physical time. Hence, shorter simulation time is needed to obtain a reliable time- and phase-averaging statistics. Such feature is important in LES cases that involve dynamic motion of vehicle, because they normally need over hundred thousand of time steps to obtain an adequate phase-averaged statistic.

In the present study, the aerodynamic quantities were estimated in each time step, with the step size $\Delta t$ $=5 \times 10^{-5} \mathrm{~s}$. To ensure numerical stability, each LES case was begin with the 1 st-order-upwind scheme for the convective term of the governing equations. Then, from step\# 10,000 (corresponds to $t=0.5 \mathrm{~s}$ ) onward, it was switched to the 2nd-order-center-differencing scheme with a blending of 5\% of 1 st-orderupwind scheme. The results obtained from the 1st-order-upwind scheme were omitted in the estimation of the aerodynamic quantities due to high numerical viscosity introduced by the scheme. In addition, in the dynamic pitching LES cases, the results obtained from the first five cycles were not use in the phaseaveraging computations. This is because the flow may still be developing in the beginning of the simulation. Concerning this, the author has checked the time series of the obtained aerodynamic properties, and confirmed that the flow is in a stable periodic condition when the phase-averaging computation began.

The length of physical time required in the dynamic pitching LES cases is depends on the number of cycle $N$ needed to obtain the reliable phase-averaged statistics. In this regard, Wernert and Favier (1999) has proposed a criterion to determine the suitable $N$. Accordingly, it was found that for model A and model $\mathrm{B}, N$ is equaled to 11 and 8 , respectively. In this paper, the presented phase-averaged quantities are the averaged over 13 pitching cycles. 


\subsection{Hardware and software}

The simulation software is an in-house CFD code "FrontFlow/red ver. 2.8", which was originally developed for the "Frontier Simulation Software for Industrial Science" project and optimized for vehicle aerodynamics simulation by Tsubokura et al. (2009a). The code has been validated successfully in the previous works (Tsubokura et al., 2009b) where good agreement is attained in the comparison between the numerical results and wind tunnel measurements for pressure distribution along the centerline of ASMO model and flow field around a full-scale production vehicle including complicated engine room and under body geometry.

We carried out the LES computations on the computing facilities of University of Tokyo. The hardware is a supercomputer developed by Hitachi Ltd., namely, HITACHI SR16000. It comprises 56 processor nodes. Each node consists of four 8-core IBM POWER7 $(3.83 \mathrm{GHz})$ processors. The total peak performance of SR16000 is 54.906 Tflops with 11200 Gbytes memory. For the present study, we used eight nodes in each LES case, which correspond to 256 parallel simulation realized by MPI (Message Passing Interface) installed in the code.

\subsection{Computational domain and boundary conditions}

The shape of the computational domain resembled a rectangular wind-tunnel test section. Its cross section covered $5 l$ upstream of the model, $13 l$ downstream, $4 w$ on both sides, and a height of $7.2 h$. The corresponding blockage ratio is $1.53 \%$, which is well within the typically accepted range of $5 \%$ in automotive aerodynamic testing (Hucho and Sovran, 1993). The whole domain comprises around 12 million numerical elements with 3 million nodes. Finer elements were constructed nearby the models to capture more details of the flow information around it (see Fig. 2). In addition, nine layers of prism mesh were generated from the surface of the models. The thickness of the first layer equals $1 \mathrm{~mm}$. As a result, the typical wall distance of the first nearest grid point is less than $y^{+}=150$, which is within the logarithmic layer of the mean velocity profile.

At the inlet boundary, the approach flow was set to be a constant, uniform velocity of $39 \mathrm{~m} / \mathrm{s}$, which corresponds to Reynolds number Re of $1.2086 \times 10^{6}$, based on the vehicle length $l$. At the outflow boundary, we imposed a zero gradient condition. We divided the ground surface into two regions. The first region which was $5.0 \mathrm{l}$ from the inlet was defined as free-slip wall boundary. This setting allows us to prevent boundary layer formation before the flow reaches the models. The remaining ground surface was treated by wall-model with the assumption of fully developed turbulent boundary layer. The ceiling and lateral boundaries of the domain were treated as free-slip wall boundary.

For the vehicle models' surface, considering that the $y^{+}$is within the logarithmic range, hence we estimated the surface friction by imposing the assumed log-law profile to the instantaneous velocity field. Although we attempted to model the boundary layer in the vicinity of vehicle surface instead of directly solving it, which might compromise the accuracy in estimating the aerodynamic forces such as drag and lift. However, the main aim of the simulation is to reproduce the flow structures that shed from the vehicle body for the investigation of physical mechanism, not to estimate precisely the aerodynamic forces. Moreover, the flow structures are mainly dictated by the geometry of vehicle and their scales are relatively large. Hence, we suppose that the wall-modeled approach is adequate for the purpose of the present study. 

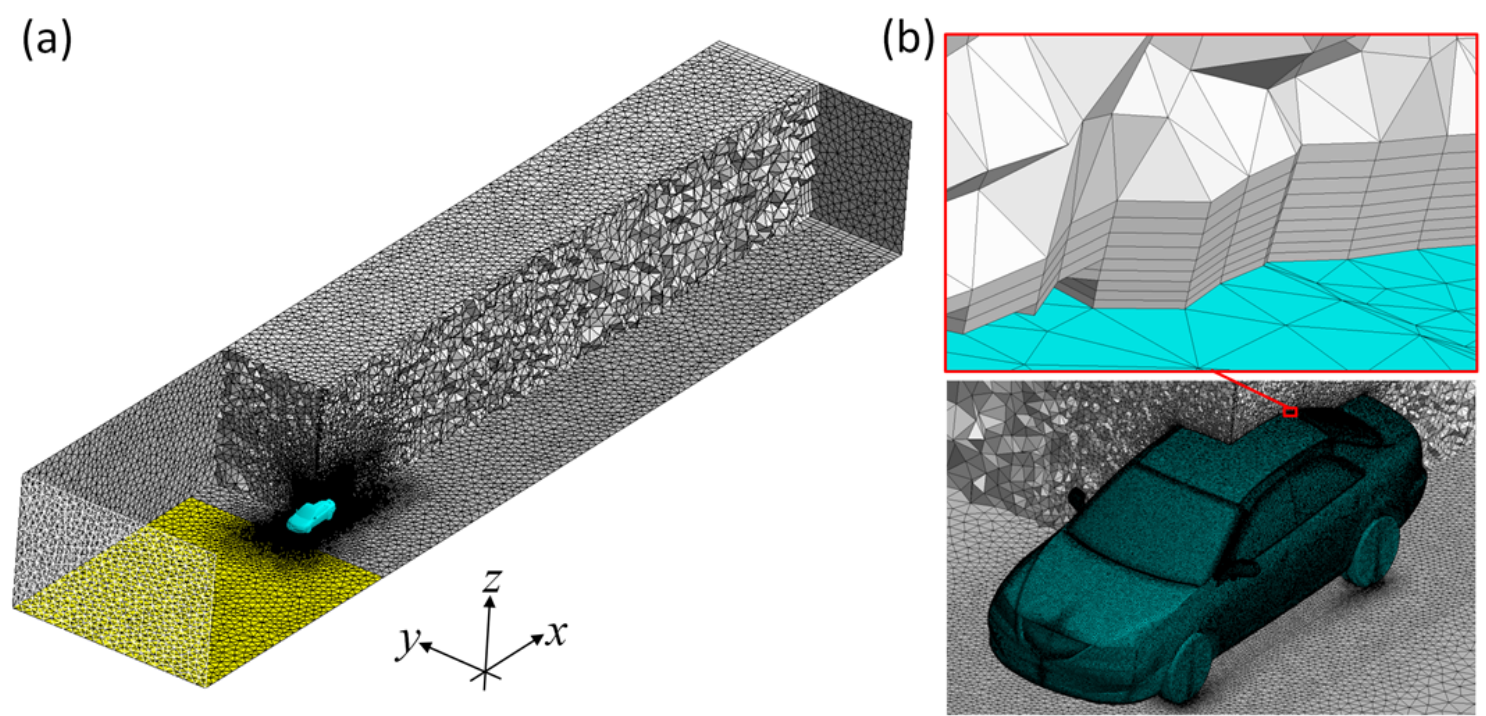

Figure 2. Numerical grid (a) Computational domain and meshes; (b) prism mesh layers

\subsection{Validation}

To validate the numerical method, we compared the flow structures obtained from the LES to the corresponding flow structures observed in the wind tunnel measurements (see Fig. 3), as well as the drag and life coefficients. The measurements were conducted in the Mazda Wind Tunnel, which has a closed test section with cross-sectional area of $6 \times 4 \mathrm{~m}$ and a length of $12 \mathrm{~m}$. For consistency of comparison, both the LES and wind-tunnel measurements were conducted at the same Re, with the vehicle fixed at zero degree pitch angle.

The test vehicle was the Vehicle-A used in the study by Okada et al [3]. It should be noted that the vehicle used in the wind tunnel measurements was the real production car, while the one used in the LES was simplified model with simple wheel geometry, exclusion of engine room, etc. Therefore, some discrepancy is to be anticipated.

As shown in Fig. 3, at the streamwise location $x=1.96 \mathrm{~m}$, the LES successfully reproduced the drop in the total pressure due to the wake of the side mirror. At the downstream locations, this wake structure expanded and faded, which implies that it has decayed as it traversed downstream. In addition, the LES has also reproduced the total pressure drop at the side of the car roof, which corresponds to the A-pillar vortex (marked "A"). Meanwhile, the $C_{d}$ and $C_{l}$ obtained from LES are in excellent agreement with the corresponding wind tunnel data. Hence, we can conclude that the numerical method is suitable for automotive aerodynamic investigations.

Table 1. Mean aerodynamic forces of the Vehicle A

\begin{tabular}{ccc}
\hline & $C_{d}$ & $C_{l}$ \\
\hline LES & 0.32 & 0.21 \\
Wind tunnel & 0.32 & 0.20 \\
\hline
\end{tabular}



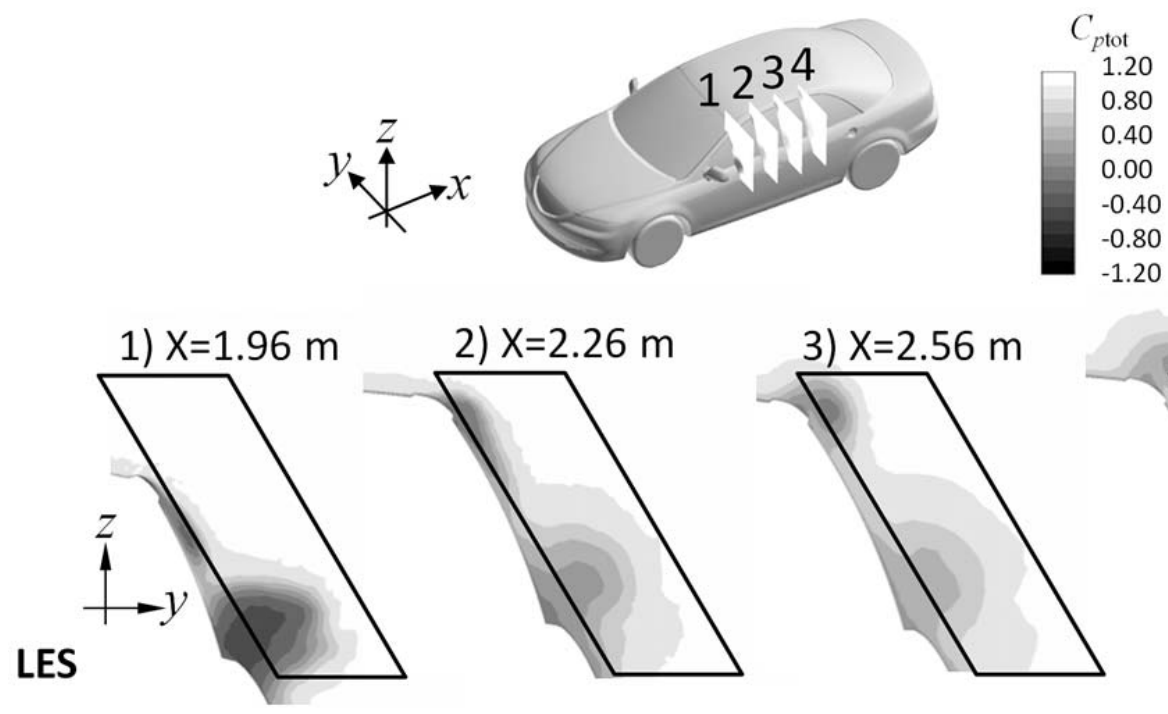

2) $X=2.26 \mathrm{~m}$

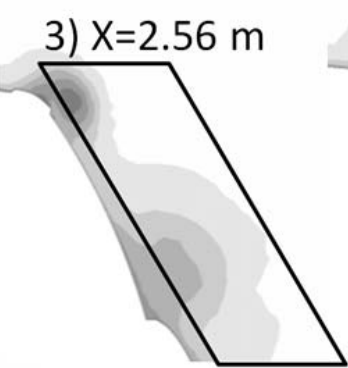

4) $X=2.86 \mathrm{~m}$
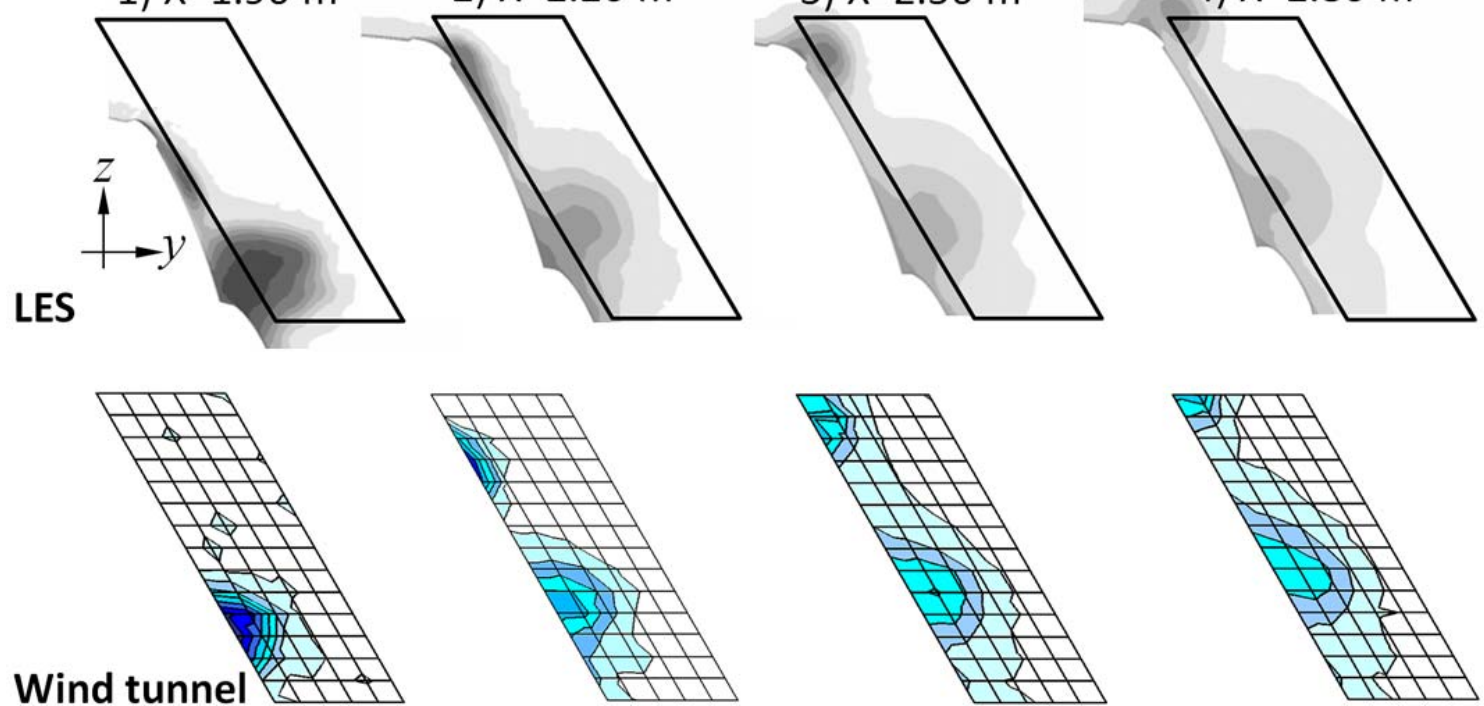

Fig. 3. Comparison of total pressure distribution between LES and wind-tunnel measurement.

\section{Formulation of Aerodynamic Damping Coefficient}

\subsection{Periodic-pitching-oscillation condition}

Alteration in the position of a vehicle due to ride-height fluctuation can cause changes in the flow structures around the vehicle. Interaction between these flow structures and the vehicle body can result in aerodynamic forces that can have the tendency to restore the stability of the vehicle or to further enhance its motion and make it unstable. To probe the stability attitude of the models, we imposed a prescribed periodic pitching oscillation on them during LES by ALE technique.

Okada et al. (2009) reported that the sedan-type vehicle with lower pitching stability has exhibited more severe ride-height fluctuation at the rear of the vehicle than at the front. Hence, we rotated the models about the front wheel axle to simulate the ride-height fluctuation type observed in the road test (see Fig. 4). The pitch angle $\theta$ was defined as $\theta=\theta_{0}-\theta_{1} \cos \phi(t)$, where, $\phi(t)=2 \pi f t$, while $\theta_{0}$ and $\theta_{1}$ were equaled to 0.9 . Therefore, the models were forced to oscillate between $0^{\circ}$ to $1.8^{\circ}$ pitch. The pitching frequency $f$ was $1 \mathrm{~Hz}$. This frequency was chosen to match the road test rear-ride-height fluctuation Strouhal number St of 0.13 (Okada et al., 2009). Phase-averaged results presented in this paper are computed over 15 cycles after the LES computation achieved a stable periodic condition.

Figure 4 shows the conventions of aerodynamic pitching moment $M$ and pitch angle $\theta$. Due to the very high computing resources required in the LES involving the ALE algorithm, we therefore employed the high-performance computing technique presented by Tsubokura et al. (2009a). 


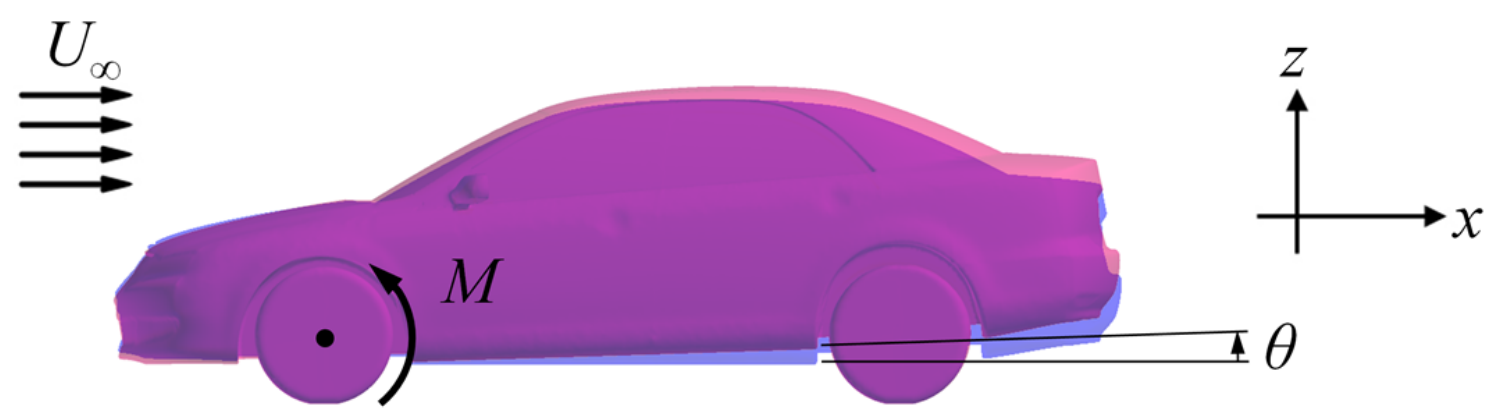

Fig. 4. Sign convention of aerodynamic pitching moment and pitch angle.

\subsection{Definition of aerodynamic damping coefficient}

The computed phase-averaged pitching moment $\langle M\rangle_{p}$ can be decomposed into the steady and unsteady components. The equation for phase-averaged pitching moment $\langle M\rangle_{p}$ in terms of pitch angle $\theta$ is given as the following expansion:

$$
<M>_{p}=C_{0}+C_{1}+C_{3} \dot{\theta}+C_{4} \ddot{\theta}
$$

where, respectively, the single dot and double dot in the third and fourth terms indicate the first and second derivatives with respect to time $t$. Both $C_{0}$ and $C_{1}$ are static components; the former denotes the pitching moment $M$ at zero pitch, while the latter describes the quasi-static behavior by taking into account the pitch-angle variation in a static manner. $C_{2}$ is associated with aerodynamic damping, and $C_{3}$ is an added moment of inertia that is proportional to angular acceleration.

Substituting the pitch angle equation into Eq. (7) and introducing new parameters $M_{\text {stat }}, M_{\text {dis }}$ and $M_{\text {ang }}$, the phase-averaged pithing moment can be rewritten as

$$
<M>_{p}=M_{\text {stat }}+M_{\text {ang }} \sin \phi(t)+M_{\text {dis }} \cos \phi(t)
$$

where, $M_{\text {stat }}$ is a constant, which set the baseline for $\langle M\rangle_{p} . M_{\text {dis }}$ is the amplitude of the term which inphase with the imposed pitching displacement, and $M_{\text {ang }}$ is the amplitude of the term in-phase with the angular velocity. We obtained these coefficients by fitting Eq. (8) to the $\langle M\rangle_{p}$ data sets by least mean square fitting.

During one pitching cycle, time $t$ varies from 0 to $2 \pi / \omega$. Hence, the work done by $M$ on the vehicle model during one pitching cycle is:

$$
W=\frac{1}{\omega} \int_{0}^{2 \pi} M \frac{\mathrm{d} \theta}{\mathrm{d} t} \mathrm{~d}(\omega t)
$$

Substituting the equations of pitch angle and phase-averaged pitching moment into Eq. (9), the work done during one pitching cycle becomes

$W=$

$$
\begin{aligned}
& M_{\text {stat }} \theta_{1} \int_{0}^{2 \pi} \cos \omega t \mathrm{~d}(\omega t)+ \\
& M_{\text {dis }} \theta_{1} \int_{0}^{2 \pi} \sin \omega t \cos \omega t \mathrm{~d}(\omega t)+M_{\text {ang }} \theta_{1} \int_{0}^{2 \pi} \cos \omega t \cos \omega t \mathrm{~d}(\omega t)
\end{aligned}
$$

The first and second integrals yield the value zero, and that the third one is $\pi$. Hence, the net work per pitching cycle is:

$$
W=M_{\text {ang }} \theta_{1} \pi
$$

The result of the integration reveals that the net work done on the vehicle by aerodynamic pitching moment $M$ over a pitching cycle is depends on the component in-phase with the angular velocity $M_{\text {ang. In }}$ Eq. (11), $\theta_{1}$ and $\pi$ are given. Hence, the parameter $M_{\text {ang }}$ reflects the dynamic response of the vehicle. This parameter can be presented in a non-dimensional form. If normalized in a similar manner to the pitching moment coefficient, it becomes: 


$$
C_{\mathrm{AD}}=\frac{M_{\mathrm{ang}}}{1 /{ }_{2} \rho U_{\infty}^{2} A l_{w}}
$$

where $\rho, U_{\infty}, A$, and $l_{\mathrm{w}}$ are fluid density, mainstream velocity, vehicle frontal area, and wheelbase. It depends on the sign of $C_{\mathrm{AD}}$, a negative value implies a tendency for aerodynamics to damp the pitching oscillation, whereas a positive value enhances the vehicle motion (i.e. negative damping). The coefficient thus enables quantitative evaluation of vehicle stability; hence, it is termed "aerodynamic-damping coefficient."

\section{Results and Discussion}

\subsection{Time-averaged, predominant flow structures}

Figure 5 shows the predominant flow structures around the upper body of the models obtained from the stationary LES cases, with the models being fixed at zero degree pitch. As depicted, the main differences in the upper body flow structures are the A- and C-pillar vortices. In model A, the strong Apillar vortices are associated with its angular A-pillar geometry. Similarly, the angular C-pillar edge in model B has generated the relatively strong C-pillar vortices.

In addition, the streamlines and surface static pressure distribution in Fig. 6 suggest that the weak Cpillar vortices in model A have allowed the flow from the sides to divert inwards above the trunk deck. As a consequence, more flow is passing over the sides of its trunk deck. We conjecture that the wide low pressure regions (indicated by the red triangles in Fig. 6) could be due to two factors: 1) the flow which is allowed to accelerate over the sides of trunk deck; 2) the widespread C-pillar vortices due to rounded Cpillar geometry; thus, wide vortex-induced, low pressure regions. 


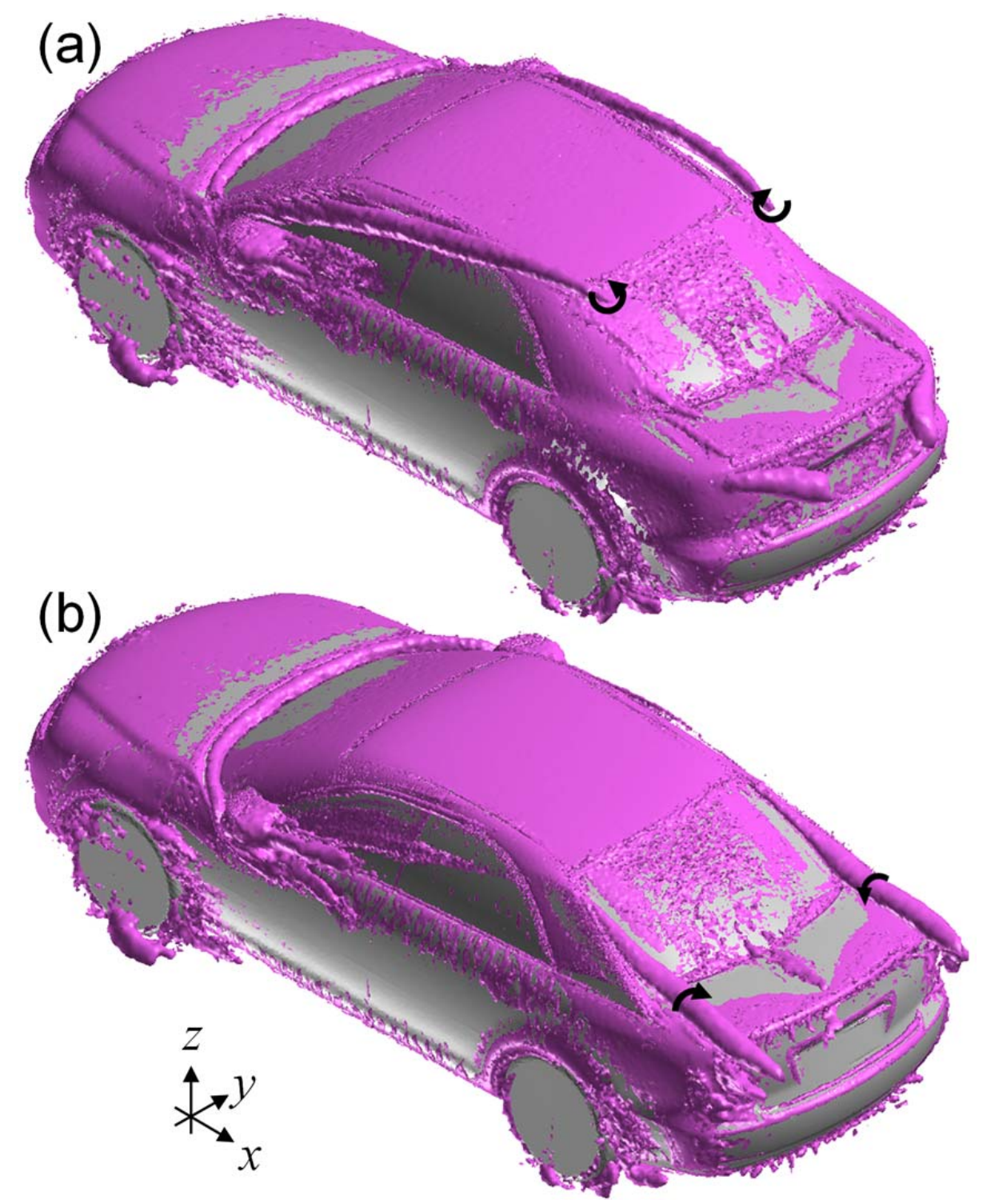

Fig. 5. Time-averaged, predominant flow structures depicted by ISO surface of pressure Laplacian $=0.03$ Pa.m ${ }^{-2}$. (a) Model A and (b) Model B. 
(a)

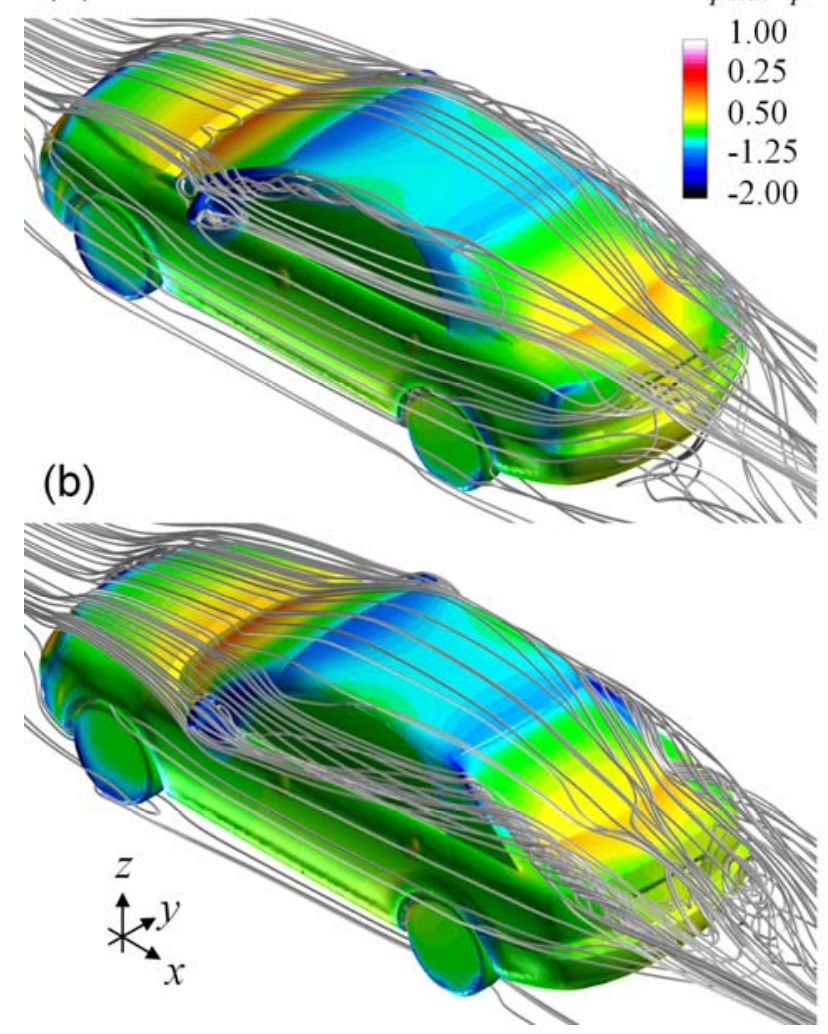

Enlarged view
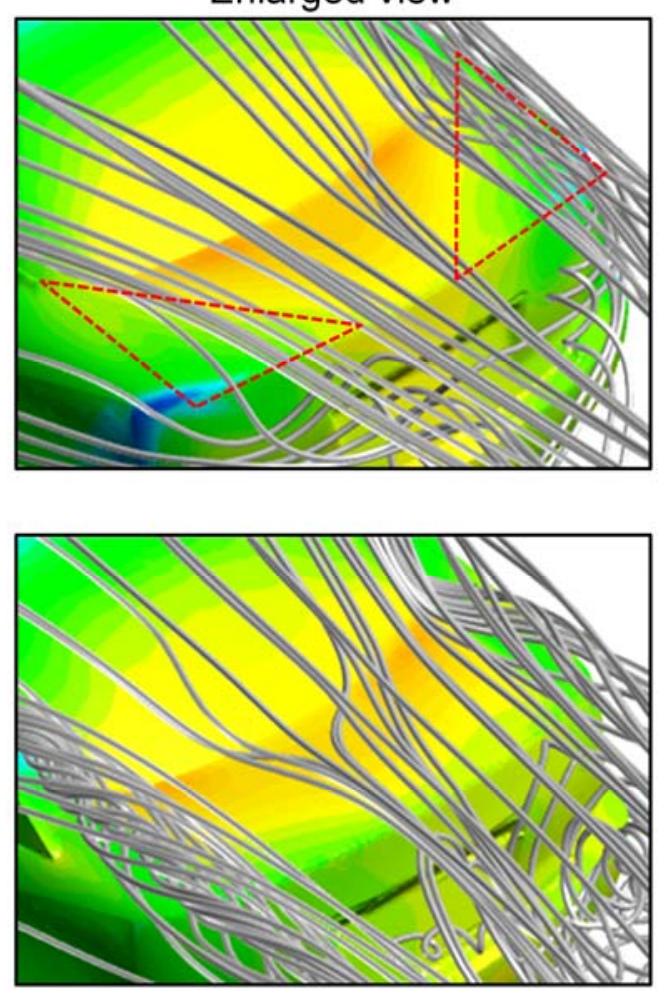

Fig. 6. Streamlines and distribution of time-averaged surface static pressure $\left.<C_{p \text { stat }}\right\rangle_{p}$ (normalized by dynamic pressure of the main stream velocity). (a) Model A and (b) Model B.

\subsection{Aerodynamic damping characteristics}

Table 2 summarizes the aerodynamic damping coefficient $C_{\mathrm{AD}}$ for the two models, as well as the contribution of each body part. As depicted, both of them have negative $C_{\mathrm{AD}}$. Hence, the work done due to aerodynamics tended to suppress the pitching motion of the models, i.e. damping of pitching instability. Between them, however, model $\mathrm{B}$ has a higher damping, by about $21 \%$. This tendency agrees with the road test results tendency in the sense that the vehicle with rounder A-pillar configuration has higher pitching stability (Okada et al., 2009).

Table 2. Overall $C_{\mathrm{AD}}$ and contribution of body parts

\begin{tabular}{ccccccc}
\hline$C_{\mathrm{AD}}\left(\times 10^{3}\right)$ & Model A & $\%$ & Model B & $\%$ & Diff. & $\%$ \\
\hline Underbody & -18.36 & 87.0 & -19.35 & 74.6 & -0.99 & 20.5 \\
Body & -1.31 & 6.2 & -1.94 & 7.5 & -0.62 & 12.9 \\
Car roof & -1.47 & 7.0 & -1.69 & 6.5 & -0.22 & 4.6 \\
Windshield & -1.37 & 6.5 & -1.25 & 4.8 & 0.12 & -2.6 \\
Rear shield & -0.60 & 2.8 & -1.47 & 5.7 & -0.88 & 18.1 \\
Deck & 0.75 & -3.6 & -0.74 & 2.8 & -1.49 & 30.9 \\
Front & 0.02 & 0.0 & 0.02 & 0.0 & 0.00 & 0.0 \\
Bonnet & 0.07 & -0.4 & 0.10 & -0.4 & 0.02 & -0.5 \\
Base & 1.18 & -5.6 & 0.40 & -1.5 & -0.77 & 16.0 \\
\hline Overall & $\mathbf{- 2 1 . 1 0}$ & $\mathbf{1 0 0 . 0}$ & $\mathbf{- 2 5 . 9 3}$ & $\mathbf{1 0 0 . 0}$ & $\mathbf{- 4 . 8 3}$ & $\mathbf{1 0 0 . 0}$ \\
\hline
\end{tabular}

The reason for the two models to have the different damping magnitudes was mainly because of the opposite trunk deck contributions. Fig. 7 shows the curves of trunk deck fitted function (solid lines) of the 
two models. In model A, the curve reaches the maximum peak during tail-up pitching cycle and minimum peak during tail-down pitching cycle. Hence, its trunk deck contribution imparted a destabilizing tendency.

\section{(a)}
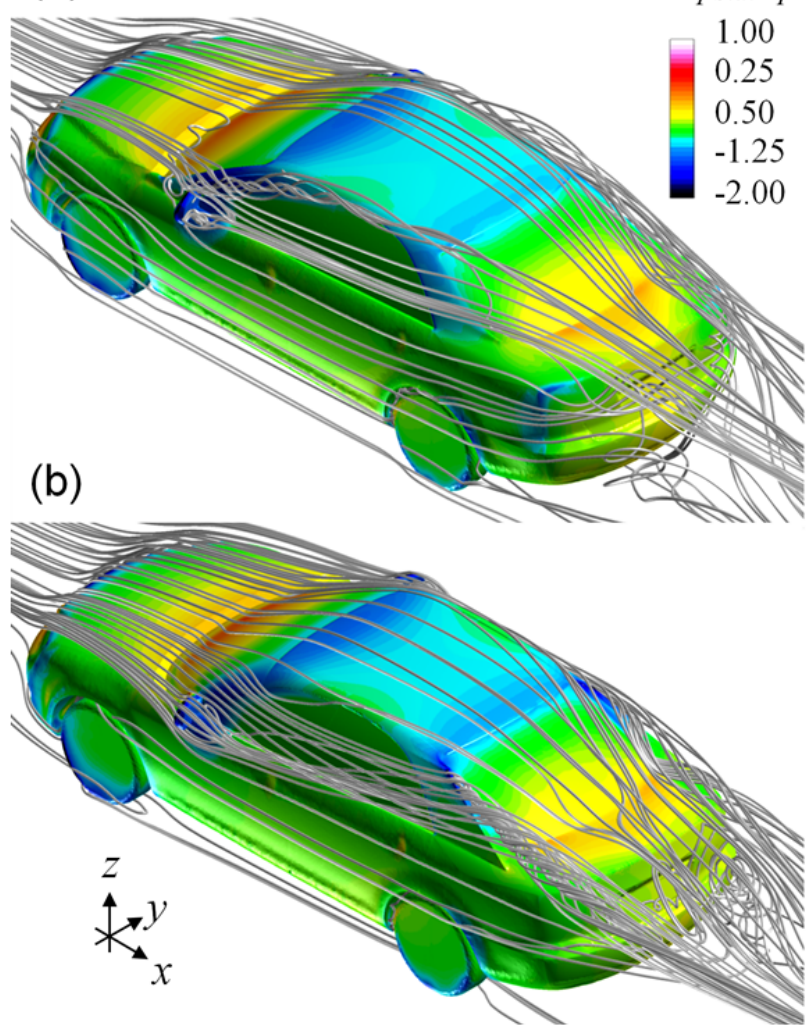
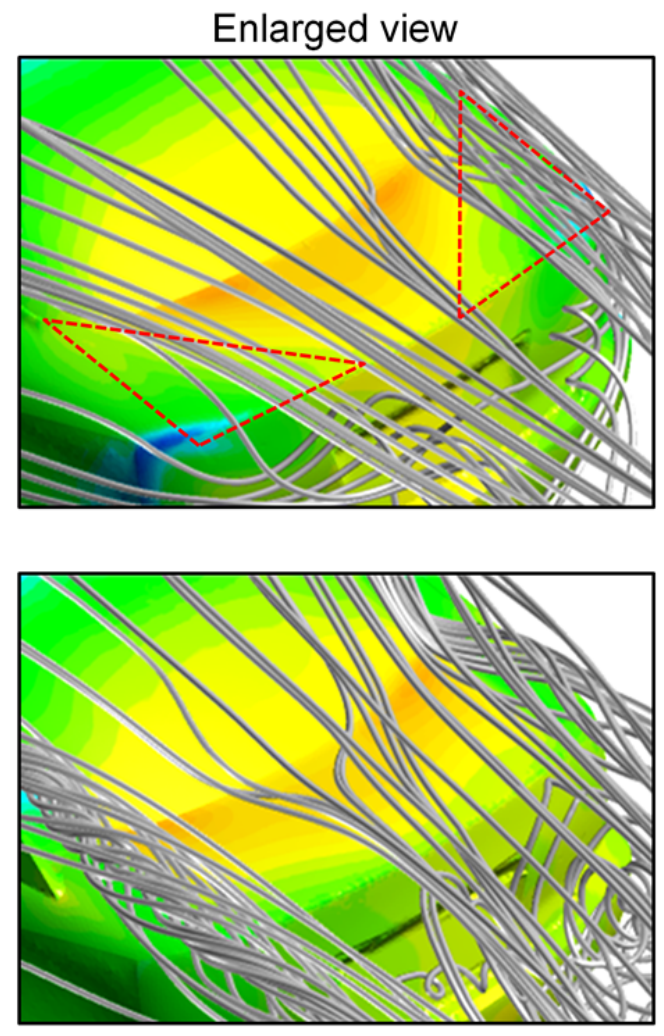

Fig. 7. Trunk deck phase-averaged $L_{\mathrm{prs}}, M$ and the corresponding fitted functions.

Fig. 7 shows that the curves of phase-averaged trunk deck pressure force $\left\langle F_{\text {prs_deck }}\right\rangle_{p}$ and pitching moment $\left\langle M_{\text {deck }}\right\rangle_{p}$ are in excellent correlation. This implies that $\left\langle M_{\text {deck }}\right\rangle_{p}$ is mainly caused by the trunk deck surface static pressure. The higher attainable $\left\langle M_{\text {deck }}\right\rangle_{p}$ in model $\mathrm{A}$ is associated with its relatively low trunk deck surface pressure, which is caused by the wide low pressure regions at the sides of its trunk deck (as has been discussed in section 5.1).

To produce a maximum damping, the pressure force has to be in-phased with the angular velocity of pitching. That is, $\left\langle F_{\text {prs_deck }}>p\right.$ to peak at $\varphi=270^{\circ}$, and reaches the minimum at $\varphi=90^{\circ}$. The $\left\langle F_{\text {prs_deck }}\right\rangle_{p}$ curve for model $\mathrm{B}$ is in line with these criteria with about $70^{\circ}$ shifting, whereas the one for model $\mathrm{A}$ shows an opposite sense. Hence, the trunk deck contributions for the two models exhibit the opposite signs.

\subsection{Transient effects on the aerodynamic properties due to pitching}

\section{oscillation}

During tail-up pitching cycle $\left(\varphi=0\right.$ to $\left.180^{\circ}\right)$, the C-pillar vortices in the two models attenuated (as may be evident from the decrease in the size of C-pillar vortices with $\theta$ in Fig. 8). This is due to the decrease in the slant angle of C-pillar edge. As a result, the trunk deck surface pressure induced by the Cpillar vortices increases with $\theta$ (marked "i" in Fig. 9). Consequently, $<M_{\text {deck }}>_{p}$ decreases.

Meanwhile, the increase in the slant angle of A-pillar edge will cause an increase in the roll up of Apillar vortices in model A. Above the trunk deck, the A-pillar and C-pillar vortices which rotate in directions opposite one another interact with each other. Their interaction generates cross flow through the clearance between them (see Fig. 8(a) and 10(a)). This effect is more pronounced at larger $\theta$ since the two vortices were brought closer together when the C-pillar vortices were elevated by the trunk deck. The strong cross flow (at larger $\theta$ ) promotes the formation of upwash inducing, circulatory structures at the central region above the trunk deck (see Fig. 10(b)). Hence, the surface pressure at the central region of trunk deck decreases with $\theta$ (marked "ii" in Fig. 9) due to relatively strong upwash. 
Therefore, in model A, the increase in the surface pressure at the sides of trunk deck (induced by Cpillar vortices) and the decrease in the surface pressure at the central region (due to upwash inducing structures) counterbalance each other. This causes the curves of $\left\langle F_{\text {prs_deck }}\right\rangle_{p}$ and $\left\langle M_{\text {deck }}>_{p}\right.$ in model A to have a more moderate slop when compared to the one for model B.

During tail-down pitching cycle $\left(\varphi=180\right.$ to $\left.360^{\circ}\right)$, the opposite occurred in regard to how the change in vehicle attitude influences the aerodynamic properties. Particularly, the strength of A- and C pillar vortices, as well as the surface pressure of trunk deck.

The discussions thus far explained only the quasi-steady behavior of the aerodynamic properties, which are depending on $\theta$. The phase-shift in $\left\langle M_{\text {deck }}{ }_{p}\right.$, however, is due to dynamic effect. In model $\mathrm{B}$, the $\left\langle M_{\text {deck }}\right\rangle_{p}$ curve reached the minimum peak before $\varphi=180^{\circ}$. This is because the trunk deck surface is at the windward side during tail-up pitching cycle. Thus, the trunk deck surface pressure increased due to the dynamic pressure of the flow. It may be conjectured that the minimum peak is at the instant when the sum of static pressure increment at the sides (due to C-pillar-vortex attenuation) and pressure increase at the central region (due to dynamic effect) is at the optimum.

During tail-down pitching cycle, the trunk deck is at the leeward side. Hence, the flow above it is being "pulled" away as the trunk deck moves downwards. Hence, the maximum peak of $\left\langle M_{\text {deck }}>_{p}\right.$ for model $\mathrm{B}$ is conjectured to be at the instant when the sum of surface pressure decrement at the sides (induced by the C-pillar-vortices) and the pressure decrement due to model motion is at the optimum.

In model $\mathrm{A}$, the $<M_{\text {deck }}>_{p}$ curve reached the minimum peak after $\varphi=180^{\circ}$. One plausible explanation to this would be that the interaction between the A- and C-pillar vortices has generated the maximum upwash in the central region, prior the maximum pitch angle. Thus impedes the pitching moment to reach the minimum peak before $\varphi=180^{\circ}$ due to the upwash-induced, low surface pressure in the central region. This would be the case if the tail-up pitching motion could enhance the roll up of A-pillar vortices. 
(a)
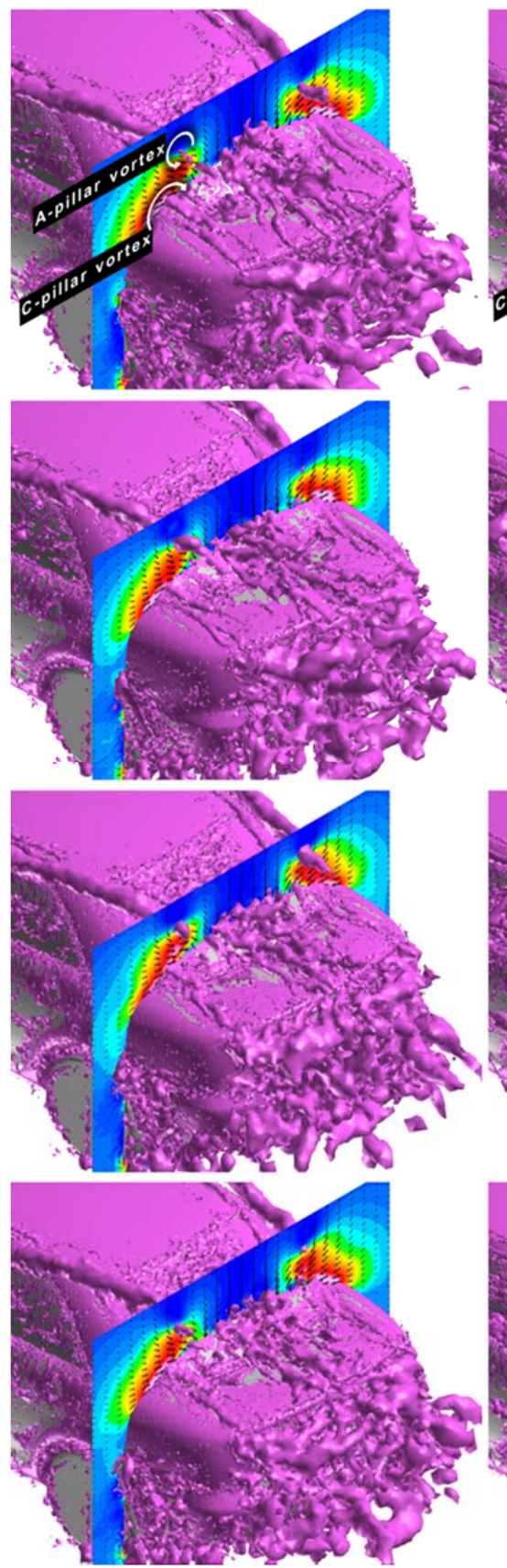

Fig. 8. Phase-averaged flow field above the trunk deck; Distribution of transverse velocity component at

$\mathrm{x} / \mathrm{l}=0.17$ from the rear end; ISO surface of pressure Laplacian $=0.013 \mathrm{~Pa} \cdot \mathrm{m}^{-2}$. (a) Model A and (b) Model B. (b)
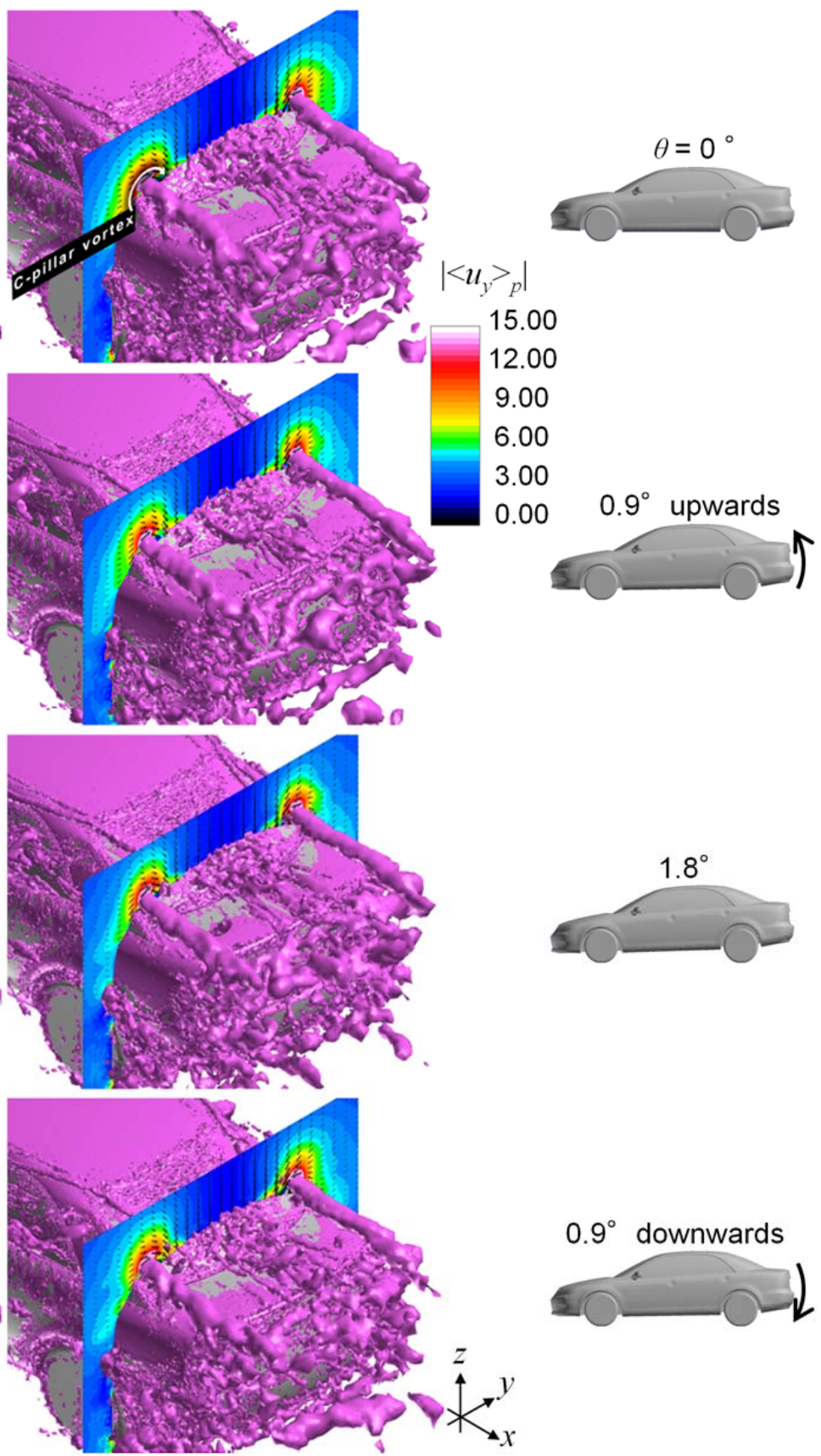

$0.9^{\circ}$ downwards

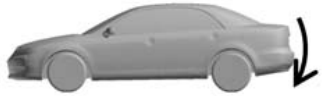


(a)

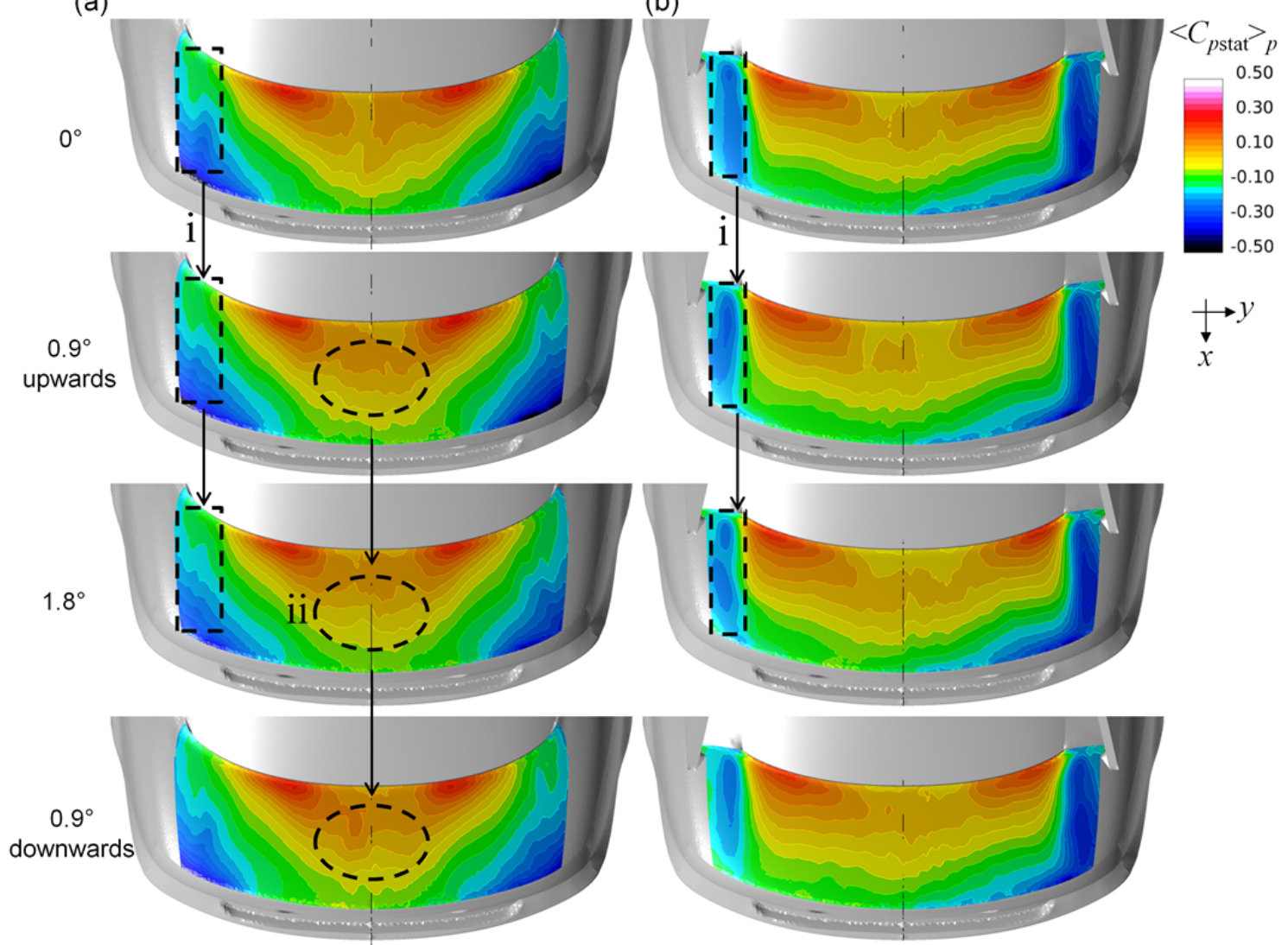

Fig. 9. Distribution of phase-averaged trunk deck surface pressure. (a) Model A and (b) Model B. 
(a)
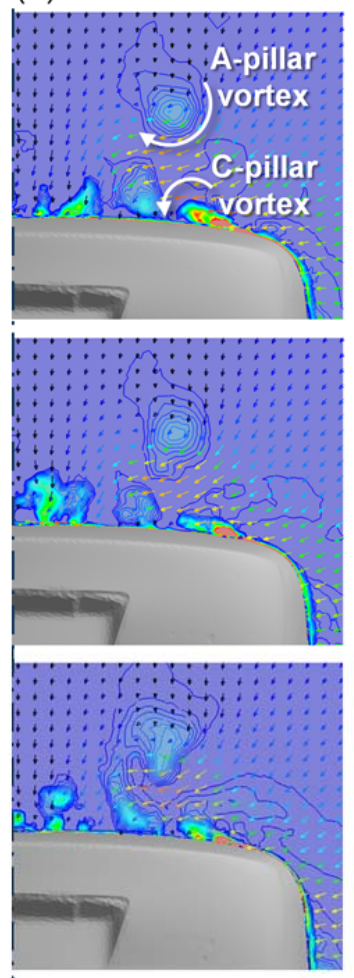

$\left.<u_{y z}\right\rangle_{p}$

0.400

0.300

0.200

0.100

0.000 (b)

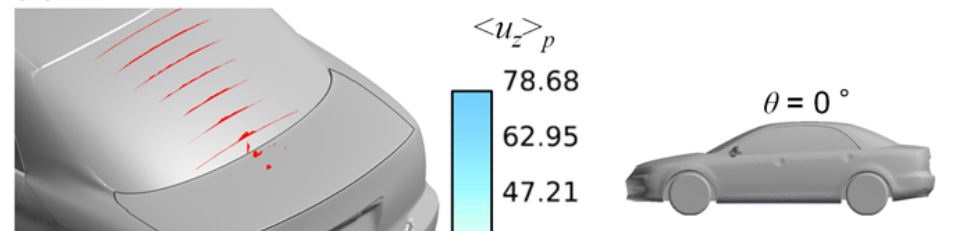

31.47

15.74

0.00
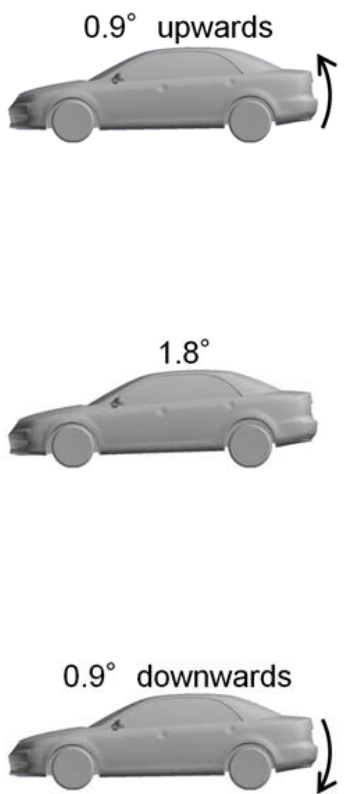

iAxis of symmetry

Fig. 10. (a) Effect of pitching on flow properties above the trunk deck (visualization plane at $x / 1=0.106$ from the rear end) and (b) distribution of phase-averaged vertical velocity component (threshold at $0 \mathrm{~m} / \mathrm{s}$ ); Model A.

\subsection{Aerodynamic damping mechanism}

The schematic diagram in Fig. 11 summarizes the mechanism of aerodynamic damping of pitching oscillation for sedan-type vehicles. In the two models, the C-pillar vortices attenuate with $\theta$. Hence, the effect of C-pillar vortices causes the surface pressure at the sides of trunk deck to increase during tail-up pitching cycle, and decrease during tail-down pitching cycle. This produces a tendency to suppress the pitching oscillation of the models - stabilizing.

In model A, the roll up of A-pillar vortices increases with $\theta$. Hence, the surface pressure at the central region of trunk deck induced by the upwash (which is associated with the A-pillar vortices) decreases during tail-up pitching cycle, and increases during tail-down pitching cycle. This produces a tendency to enhance the pitching oscillation of model A - destabilizing.

We obtained negative $C_{\mathrm{AD}}$ in the two models, i.e. aerodynamically stable. Hence, this implies that the destabilizing tendency caused by the A-pillar effect in model A, has lesser impact than the stabilizing tendency caused by the C-pillar effect. 


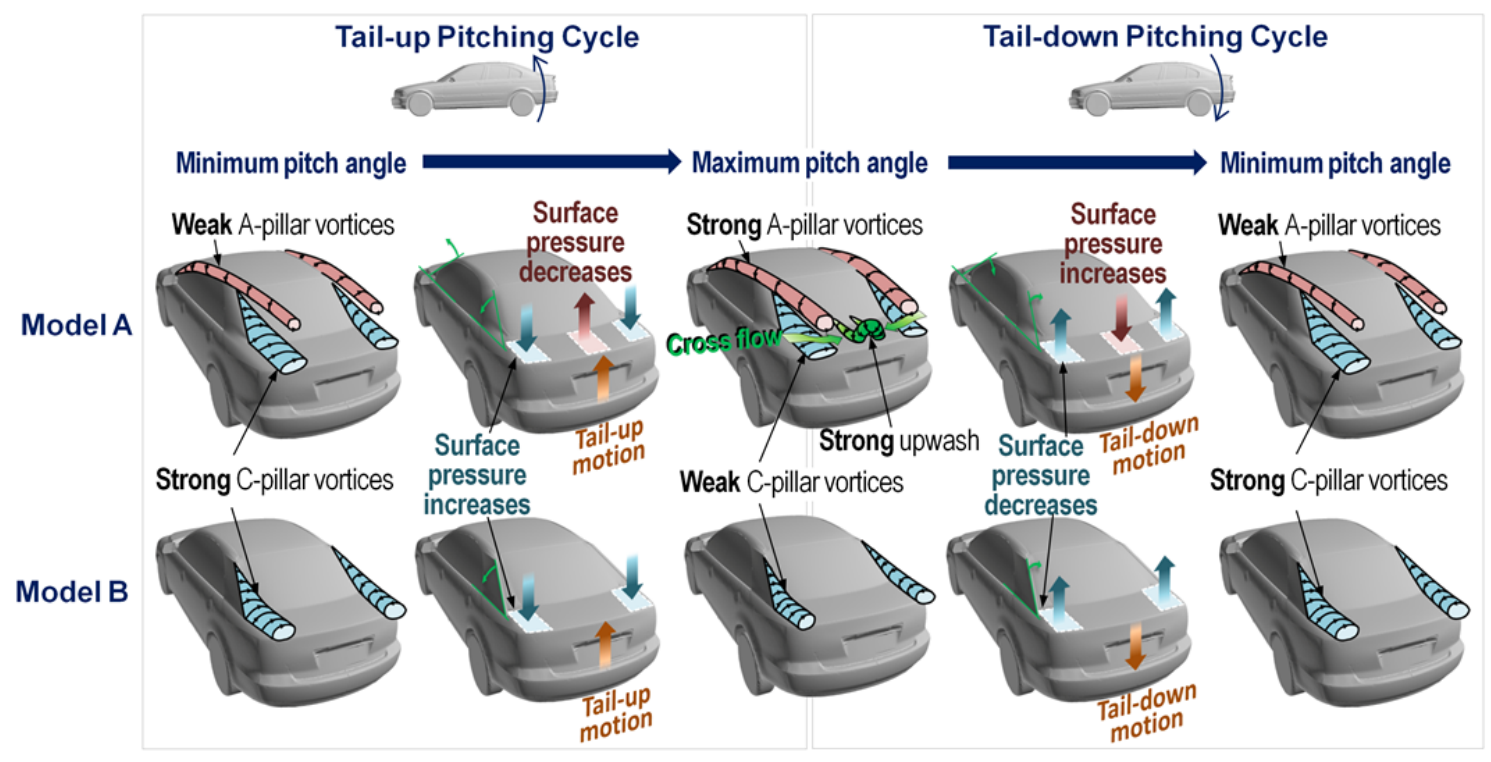

Fig. 11. Mechanism of aerodynamic damping of pitching oscillation.

\subsection{Generalizability of simple body model results to automotive aerodynamics}

Cheng et al. (2012) reported that the simple body model with rounded A-pillar and angular C-pillar exhibited a higher aerodynamic damping, by the difference about $41 \%$. In the present study, the basic sedan models have also exhibited the same tendency. The smaller difference in $C_{\mathrm{AD}}$ in the case of basic sedan models $(21 \%)$ could be attributed to the relatively small pitching amplitude adopted in the present study. Its value is determined by the ride-height-variation range a sedan-type vehicle would experience in real driving situations (based on the road test results of the study by Okada et al., 2009).

In addition, the difference in $C_{\mathrm{AD}}$ between the two simple body models is mainly caused by the trunk deck contribution (Cheng et al., 2012). The factors that influenced the trunk deck surface pressure during pitching oscillation are the roll up of C-pillar vortices which induced the relatively low static pressure region at the sides of trunk deck, and the interaction between the A- and C-pillar vortices (in the model with angular A-pillar configuration) which promote the formation of upwash in the central region.

In the present study, the basic sedan models have also exhibited the low pressure regions at the sides of trunk deck (marked "i" in Fig. 9), which is caused by the C-pillar vortices. Similar to the case of simple body models (Cheng et al., 2011), the size of these low pressure region decreases with pitch angle due to the attenuation of C-pillar vortices. Meanwhile, in model A, the surface pressure in the central region of the trunk deck is also affected by the interaction between the A- and C-pillar vortices. Hence, the similar tendency shown in the results of basic sedan models and simple body models implies that the aerodynamic damping mechanism obtained from simple bluff bodies can be generalized to real vehicle aerodynamics.

\section{Conclusion}

The present study investigated the aerodynamic pitching stability mechanism of sedan-type vehicle by a large eddy simulation method. The influence of upper body geometry, particularly the effect of A- and C- pillar geometrical configurations had been examined. In addition, two vehicle models with the basic sedan-type automobile shape were used to verify the extent to which the results obtained from the generic, simple bluff body models can be applied to real vehicle aerodynamics.

For the basic sedan models investigated, the flow properties above the trunk deck were found to have the main influence on the aerodynamic pitching stability characteristic. These flow properties were dictated by the vortices shed from the A- and C-pillar edges. Particularly, the vortex shed from the Apillar edge tended to enhance the pitching oscillation of vehicle, i.e. destabilizing tendency. Whilst, the vortex shed from the C-pillar edge tended to suppress the oscillation, i.e. stabilizing tendency. 
In general, similar mechanism of aerodynamic damping of pitching oscillation was found in the cases of simple body model and basic sedan model. Hence, the use of simple bluff body in the modeling of automotive aerodynamics is deemed possible.

\section{Acknowledgments}

This work was supported by Industrial Technology Research Grant Program in 2007 from New Energy and Industrial Technology Development Organization (NEDO) for the development of FrontFlow/red-Aero; "Revolutionary Simulation Software (RSS21)" by the Ministry of Education, Culture, Sports, Science, and Technology (MEXT) for the development of FrontFlow (base code of FrontFlow/red-Aero); Japan Society for the Promotion of Science (JSPS) Grant-in Aid for Scientific Research (KAKENHI) (C) Number 23560179 for the physical mechanism of trunk-deck flow and damping; the grant for "Strategic Program-Preliminary Research Field No. 4: Next-Generation Industrial Innovations" from the Ministry of Education, Culture, Sports, Science, and Technology (MEXT)'s "Development and Use of Advanced, High-Performance, General-Purpose Supercomputers Project"; and partly supported by the National Research Foundation (NRF) grant funded by the MEST of Korea (No. 2008-0060153). This work was carried out in partnership with the University of Tokyo for the improvement of FrontFlow/red-Aero for HPC simulation.

\section{References}

Amsden, A.A., Harlow, F.H., 1970. A simplified MAC technique for incompressible fluid flow calculations. J. Comput. Phys. 6, 322-325.

Aschwanden, P., Müller, J., Knörnschild, U., 2006. Experimental study on the influence of model motion on the aerodynamic performance of a race car. SAE Paper 2006-01-0803.

Cheng, S.Y., Tsubokura, M., Nakashima, T., Nouzawa, T., Okada, Y., 2011. A numerical analysis of transient flow past road vehicles subjected to pitching oscillation. J. Wind Eng. Ind. Aerodyn. 99, 511-522.

Cheng, S.Y., Tsubokura, M., Nakashima, T., Okada, Y., Nouzawa, T., 2012. Numerical quantification of aerodynamic damping on pitching of vehicle-inspired bluff body. J. Fluid Struct. 30, 188-204.

Davenport, A.G., 1988. The response of tension structures to turbulent wind: the role of aerodynamic damping. In: Proceedings of first international Oleg Kerensky memorial conference on tension structures, the Institution of Structural Engineers. London, pp. 20-22.

Daw, D.J., Davenport, A.G., 1989. Aerodynamic damping and stiffness of a semi-circular roof in turbulent wind. J. Wind Eng. Ind. Aerodyn. 32, 83-92. 0651 .

Howell, J., Le Good, G., 1999. The influence of aerodynamic lift on high speed stability. SAE Paper No 1999-01-

Hucho, W-H., Sovran, G., 1993. Aerodynamics of road vehicles. Annu. Rev. Fluid Mech. 25, 485-537.

Hirt, C.W., Amsden, A.A., Cook, J.L., 1974. An Arbitrary Lagrangian-Eulerian Computing Method for All Flow Speeds, J. Comput. Phys. 14, 227-253.

Macdonald, J., 2002. Separation of the contributions of aerodynamic and structural damping in vibrations of inclined cables. J. Wind Eng. Ind. Aerodyn. 90, 19-39.

Marukawa, H., Kato, N., Fujii, K., Tamura, Y., 1996. Experimental evaluation of aerodynamic damping of tall buildings. J. Wind Eng. Ind. Aerodyn. 59, 177-190.

Momomura, Y., Marukawa, H., Okamura, T., Hongo, E., Ohkuma, T., 1997. Full-scale measurements of windinduced vibration of a transmission line system in a mountainous area. J. Wind Eng. Ind. Aerodyn. 77, 241-252.

Nakashima, T., Tsubokura, M., Nouzawa, T., Nakamura, T., Ichimiya, M., 2009. Flow Structures above the trunk deck of sedan-type vehicles and their influence on high-speed vehicle stability, 2nd report: Numerical investigation on simplified vehicle models using large-eddy simulation. SAE International Journal of Passenger Cars; Mechanical Systems. 2-1, 157-167.

Okada, Y., Nouzawa, T., Nakamura, T., Okamoto, S., 2009. Flow structure above the trunk deck of sedan-type vehicles and their influence on high-speed vehicle stability 1st report: On-Road and Wind-Tunnel Studies on Unsteady Flow Characteristics that Stabilize Vehicle Behavior. SAE Paper No 2009-01-0004.

Sinclair, F.M., 1994. Aerodynamic damping on offshore installations - a comparison of experimental measurements with theory. J. Wind Eng. Ind. Aerodyn. 52, 321-344.

Smagorinsky, J., 1963. General circulation experiments with primitive equations. Monthly Weather Rex. 91-3, 99-164.

Tsubokura, M., Nakashima, T., Kitoh, K., Sasaki, Y., Oshima, N., Kobayashi, T., 2009a. Development of an Unsteady Aerodynamic Simulator Using Large-Eddy Simulation Based on High-Performance Computing Technique. SAE International Journal of Passenger Cars; Mechanical Systems. 2-1, 168-178.

Tsubokura, M., Kobayashi, T., Nakashima, T., Nouzawa, T., Nakamura, T., Zhang, H., Onishi, K., Oshima, N., 2009b. Computational visualization of unsteady flow around vehicles using high performance computing. Comput. Fluids. 38, 981-990.

Van Driest, R., 1956. On turbulent flow near a wall. J. of Aero. Sci. 23, 1007-1011.

Virlogeux, M., 1998. Cable vibrations in cable-stayed bridges. In: Proceedings of the International Symposium on Advances in Bridge Aerodynamics, Copenhagen, Balkema, pp. 213-233.

Watanabe. Y., Isyumov. N., Davenport, A.G., 1997. Empirical aerodynamic damping function for tall buildings. J. Wind Eng. Ind. Aerodyn. 72, 313-321. 
Wernert, P., Favier, D., 1999. Considerations about the phase averaging method with application to ELDV and PIV measurements over pitching airfoils. Exp. Fluids. 27, 473-483. 